\title{
ACCIDENTES LABORALES POR RESIDUOS PUNZOCORTANTES EN EL PERSONAL DE SALUD: UN ESTUDIO EXPLORATORIO
}

OCCUPATIONAL ACCIDENTS BY WASTE SHARP DEVICES IN HEALTH CARE WORKERS: AN EXPLORATORY STUDY

Recibido 3 de febrero 2015 Aceptado 10 de marzo 2015

Correspondencia:

Danae Jiménez Martínez Juan Badiano No I, Col. Sección XVI Del. Tlalpan, México D.F. CP. 14080 Tel. (0155) 55732911 Ext. 1225 dana821104@hotmail.com

\section{Autores:}

Danae Jiménez-Martínez

Licenciada en Enfermería. Enfermera Adscrita del Comité de Control de Infecciones Nosocomiales. Instituto Nacional de Cardiología Ignacio Chávez.

Imelda Flores-Montes

Licenciada en Enfermería. Jefe de Enfermeras del Comité de Control de Infecciones Nosocomiales. Instituto Nacional de Cardiología Ignacio Chávez

Micaela Ordiano-Ramírez

Enfermera Especialista en Enfermería Infantil. Enfermera Adscrita del Comité de Control de Infecciones Nosocomiales. Instituto Nacional de Cardiología Ignacio Chávez

Gustavo Atescatenco-Pineda

Enfermero Especialista en el Adulto en Estado Crítico. Enfermero Adscrito del Comité de Control de Infecciones Nosocomiales. Instituto Nacional de Cardiología lgnacio Chávez

Mayeli Cervera-Rojo

Maestría en Terapia de Heridas, Estomas y Quemaduras. Enfermera Adscrita de la Clínica de heridas del Instituto Nacional de Cardiología Ignacio Chávez.

Palabras clave: Residuos punzocortantes, salud laboral, personal de salud, accidentes laborales.

Key Words: waste sharps, health workplace, personal health, work-related accidents. 


\section{RESUMEN}

Introducción. Los trabajadores de la salud están expuestos a diversos riesgos ocupacionales, entre los que destacan los accidentes por residuos punzocortantes (RP); estos desechos son fuente potencial de enfermedades infectocontagiosas y su reporte y seguimiento del trabajador es importante para evaluar su salud. El comité de infecciones nosocomiales (CIN) registra los accidentes por RP para conocer factores que desencadenaron la exposición al riesgo.

Objetivo. Describir los accidentes laborales por RP del trabajador de la salud.

Material y métodos. Estudio descriptivo, retrospectivo y transversal de enero 2012 a diciembre 2013. Muestra no probabilística $(N=63)$ incluyó trabajadores de la salud que reportaron accidentes por RP al CIN. Datos recolectados mediante cédula ex profeso de registro de punciones y lesiones accidentales, con variables: fecha del accidente y del reporte, datos del paciente, ocupación, lugar donde ocurrió, características del punzocortante, tipo de lesión, descripción del accidente y seguimiento. Datos analizados en el programa Excel con estadística descriptiva.

Resultados. Los accidentes se presentan con mayor frecuencia en turno matutino (62\%) y en el personal de Enfermería (71\%), reportándose el mismo día del evento (50\%). Se presentan más en el área de hospitalización (23\%) y unidad coronaria (19\%); 70\% dentro de la habitación del paciente. El accidente fue con aguja (44\%) y lanceta (22\%). A los 12 meses del accidente, el 3\% concluyó seguimiento.

Conclusiones. Los registros de accidentes laborales son más frecuentes en el personal de enfermería, durante el cuidado de la persona en su habitación, principalmente ocasionados por agujas. Esto conlleva a implementar estrategias que mejoren las medidas de bioseguridad y favorezcan el reporte de accidentes laborales.

Palabras clave: residuos punzocortantes, salud laboral, personal de salud, accidentes laborales.

\section{ABSTRACT}

Introduction. Health workers are exposed to various occupational risks, including accidents by waste sharp devices (WSD). These are a potential source of infections or contagious diseases, the reporting and monitoring of a worker is important in order to assess his/her health. The Committee on nosocomial infections (CIN) registers WSD accidents to known factors that triggered the exposure to risk.

Objective. Describe workplace accidents by WSD of health workers

Material and methods. Descriptive, retrospective and transversal study of January 2012 to December 2013. Non probabilistic sample $(N=63)$ included health workers who reported accidents for WSD to the CIN. Data collected by identity indifferent records of punctures and accidental injury with variables: date of the accident and report, data from the patient, occupation; place where occurred, the stab, lesion type, description of the accident and monitoring features. Data analyzed in the program Excel with descriptive statistics.

Results. Accidents occur most frequently in morning shift (62\%) to nurses (71\%), reporting the same day of the event (50\%). It happens more frequently in the area of hospitalization (23\%) and coronary care unit (19\%); $70 \%$ within the patient's room. The common accident is with a needle (44\%) and lancet (22\%). At 12 months of the accident, 3\% finished the monitoring

Conclusions. Records of occupational accidents are more frequent in the nursing staff, for the care of the person in his/her room, mainly caused by needles. This leads to implement strategies that improve biosecurity measures and encourage the reporting of accidents.

Key words: waste sharps, health workplace, personal health, work-related accidents.

\section{6}

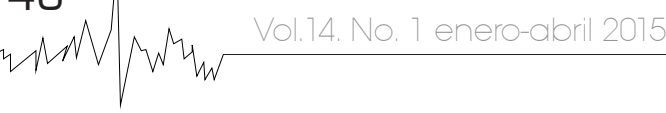




\section{INTRODUCCIÓN}

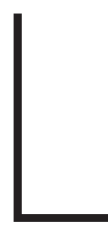

os trabajadores de la salud (TS) están expuestos a múltiples riesgos ocupacionales, entre los que predominan los accidentes por residuos punzocortantes (RP), secundario - la manipulación de los dispositivos invasivos por la atención que se brinda al usuario en una institución de salud (IS), estos desechos son fuente potencial de enfermedades infectocontagiosas, por lo que el reporte y seguimiento del trabajador es muy importante para tomar medidas oportunas.

Las IS generan diariamente materiales de desecho, Ilamados Residuos Peligrosos Biológico-Infecciosos (RPBI) que se consideran un riesgo potencial para quienes los manipulan por el peligro que representa, desde los años 80's debido a los hallazgos de residuos hospitalarios en zonas pobladas y la creciente epidemia de infecciones como el $\mathrm{VIH}$ (virus de inmunodeficiencia humana), organismos internacionales como el Centers for Disease Control (CDC), la Organización Mundial de la Salud (OMS) y la Organización Panamericana de la Salud (OPS) han emitido recomendaciones para su manejo, mismas que han sido adoptadas en la reglamentación y las políticas internas de cada país. De acuerdo a la OMS, las IS de Latinoamérica producen diariamente en promedio $3 \mathrm{Kg}$ de residuos por cama, de los que sólo del 10\% al 25\% tienen características de peligrosidad.

En México se cuenta con la Norma Oficial Mexicana NOM-087-ECOL-SSA 1-2002, Protección Ambiental - Salud Ambiental - Residuos peligrosos biológico - infecciosos -Clasificación y especificaciones de manejo, que regula el adecuado manejo de RP e incluye el concepto de actividad técnica operativa e involucra la manipulación, transporte, tratamiento y disposición final de estos.' La mayor parte de los accidentes que ocurren por RPBI es por la inadecuada manipulación de RP considerándose éste como todo objeto que tiene la capacidad de penetrar y/o cortar tejidos humanos facilitando el desarrollo de una infección, tales como agujas, alambres, tornillos, hojas de bisturí, cánulas, tubos de vidrio, adaptadores de equipos de infusión, navajas, porta, cubre objetos, lancetas, entre otros. ${ }^{2}$

El volumen y la ruta de inoculación percutánea ante la lesión producida por RP ocasionan pérdida de la integridad de la piel, poniendo en contacto al TS con fluidos orgánicos potencialmente infecciosos e influyen sobre el riesgo de transmisión del padecimiento. La inoculación percutánea de sangre implica el mayor riesgo de transmisión como el caso de VIH, que se clasifica en alto, mediano y bajo riesgo (Cuadro I). De acuerdo a la literatura, las enfermedades con mayor riesgo de transmisión y que tienen más importancia son las producidas por los virus de hepatitis B (VHB), virus de hepatitis $C(\mathrm{VHC})$ y $\vee \mathrm{IH}^{\top}$, por eso se hace énfasis en el adecuado manejo de los RP (Cuadro II).

La OMS estima que a nivel mundial existen 35 millones de TS, de los cuales aproximadamente 3 millones han experimentado anualmente un accidente por RP con patógenos sanguíneos, de los cuales 2 millones corresponden a VHB, 0.9 millones a VHC y 170, 000 a VIH. ${ }^{?}$

Cuando existe una exposición con el VIH, el 50 a 80\% de los pacientes presenta el Sindrome Retroviral Agudo (SRA), el cual se produce entre 1 a 6 semanas post entrada del virus. El antígeno p24 del core viral (antigenemia) se detecta coincidiendo con la aparición de los síntomas hasta la seroconversión (anti core p24), que ocurre entre la semana 2 y 12 post entrada del VIH. La infección tiene un período de latencia clínica que dura en promedio de 8-10 años, y concluye con las enfermedades oportunistas propias del síndrome de inmunodeficiencia adquirida. ${ }^{3}$

El VHB pertenece a la familia de los hepadnavirus de $42 \mathrm{~nm}$ y tiene una mayor incidencia en drogadictos, homosexuales, personal de salud, inmunosuprimidos y hemofílicos con un periodo de incubación de 45 a 160 días y su transmisión es por actividad sexual, parenteral o perinatal. Mientras que el VHC pertenece a la familia de flavivirus 
y se transmite con mayor frecuencia por vía parenteral a través de sangre o sus productos y en menor porcentaje sexual. Su mayor incidencia es en drogadictos, personas transfundidas y trabajadores de la salud y el periodo de incubación es de 2 a 12 semanas. ${ }^{4}$

En términos de morbilidad se tienen pocos reportes de las IS acerca de los accidentes con RP, aunque el impacto en México es relevante, ya que existe evidencia que el personal que ha sufrido un accidente con RP presentó seroconversión a VHB y $\mathrm{VHC}$, pero ninguno con $\mathrm{V} \mathrm{H}^{5}{ }^{5}$

Dentro del área hospitalaria la aplicación de conocimientos, técnicas y equipamiento para prevenir la exposición a agentes potencialmente infecciosos se conoce como bioseguridad hospitalaria, donde el objetivo es implementar medidas organizativas para contener, manipular, confinar y reducir el riesgo a la exposición potencial de agentes infecciosos al TS. Para esto se basa en principios como la universalidad, el uso de barreras y el tener los medios adecuados de eliminación de material contaminado.?

En la NORMA Oficial Mexicana NOM-087-ECOLSSA 1-2002 hay diferentes clasificaciones de acuerdo a la producción RPBl; el Instituto Nacional de Cardiología (INC) se encuentra en nivel III por generar más de 100kg al mes con un periodo de almacenamiento no mayor a 7 días.' Es importante mencionar que cuenta con políticas para el manejo de RPBI y el Comité de Control de Infecciones Nosocomiales (CCIN) es el encargado de vigilar su aplicación mediante un programa preventivo y de seguimiento del personal que ha tenido un accidente por RP, el cual consiste en 1) lavar la herida de forma inmediata, 2) notificar al jefe inmediato del personal de salud, 3) se notifica y registra el accidente en el CCIN y se da apertura del protocolo de seguimiento iniciando con una entrevista para conocer los factores que desencadenaron la exposición al riesgo, posteriormente se envía al personal de salud al laboratorio central para toma de muestras basales que incluye detección de VHB, VHC y VIH con un seguimiento a los tres meses, seis meses y al año de pruebas serológicas. Cuando el accidente ocurre con un paciente que se conoce o se sospecha de probable portador de $\mathrm{VIH}, \mathrm{VHB}$ o VHC, se realiza una valoración por el médico infectólogo, y si es necesario, se inicia tratamiento, sin olvidar que todos los pacientes son potencialmente infectocontagiosos. ${ }^{6}$

En el INC se ha observado que los accidentes de mayor prevalencia que se presentan en el TS es durante la atención hospitalaria se debe a las punciones por RP, los riesgos de una complicación depende de las funciones que realicen. El profesional de enfermería está especialmente en riesgo y es el grupo más representativo en estas situaciones, probablemente por ser quien pasa más horas en la atención directa del paciente realizando técnicas de riesgo; sin embargo, existen otras personas que también se encuentran expuestos, como son los médicos, el personal de intendencia, de laboratorios, entre otros. Aun cuando existe un protocolo de registro y seguimiento, no todo el personal que sufrió un accidente lo concluye y dada la magnitud del problema y la poca evidencia existente en nuestro medio, el presente trabajo tuvo la finalidad de describir los accidentes laborales por RP en el personal de salud del INC?

La exposición a patógenos transmitidos por la sangre producida por lesiones de agujas u otros instrumentos punzocortantes es un problema serio pero puede prevenirse, por lo que con base en los resultados el CCIN podrá analizarse el problema, implementar estrategias para concientizar al personal y disminuir los accidentes por RP.

\section{8}

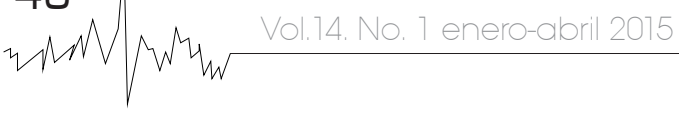




\section{MATERIAL Y MÉTODOS}

S

e realizó un estudio descriptivo, retrospectivo y transversal de enero de 2012 a diciembre de 2013. La población de estudio ( $N=63$ ) fue todo el personal que acudió al CCIN para reportar un accidente con RP. La recolección de datos se realizó mediante la cédula ex profeso de registro de punciones y lesiones accidentales con RP, que contiene datos para el análisis del riesgo al que se ha expuesto el personal de salud, así como los datos del paciente, la identificación del personal de salud, fecha del accidente y del reporte, turno, categoría, servicio, lugar físico donde ocurrió la herida, características del objeto punzocortante que ocasiona la lesión, tipo de lesión que genera, lugar anatómico de la lesión, descripción breve del accidente, valoración por el médico infectólogo de acuerdo a los factores de riesgo y seguimiento de toma de muestras durante un año.

El análisis de los datos se realizó en el programa Excel a través de estadística descriptiva con frecuencias y porcentajes para variables nominales y ordinales, así como medidas de tendencia central y de dispersión para variables cuantitativas (media, mediana, moda, cuartiles y desviación estándar).

De acuerdo a la Ley General de Salud en materia de investigación para la salud en su artículo 16,8 el presente estudio se cataloga como investigación sin riesgo, ya que sólo se recolectaron datos estadísticos de los registros clínicos del personal de salud sin revelar la identidad, por lo que no se requiere de un consentimiento informado y no se realizará ninguna intervención o modificación intencionada en las variables fisiológicas, psicológicas o sociales de los participantes. Se guardará la confidencialidad de los datos obtenidos por la institución apegándose a lo establecido en el código de Nuremberg.?

\section{RESULTADOS}

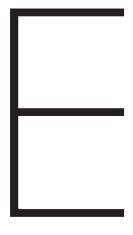

I turno con mayor frecuencia en reportar accidentes por punción fue el turno matutino (67.9\%); el 50.8\% de los accidentes se reportaron el mismo día del evento, 37.7\% lo realizó de 1-2 días después, pero el 1.6\% lo hizo después de 4 días de sucedido el accidente. De acuerdo al área de adscripción, el 69.8\% del personal está adscrito a la Dirección de Enfermería; (Cuadro III). El lugar donde ocurren con mayor frecuencia los accidentes son las áreas de unidades de cuidados críticos (30.1\%), hospitalización (22.2\%) y la sala de operaciones (9.5\%).

Con relación al lugar físico donde ocurrió el accidente, el 69.8\% fue dentro de la habitación del paciente y $11.1 \%$ en la sala de operaciones, pero llama la atención que en el 9.5\% de los casos fue en otras áreas comunes como el pasillo, la central de enfermeras y el área de lavado de material quirúrgico. El personal de salud que sufrió el accidente estaba recolectando muestras sanguíneas (44.5\%), suturaba un órgano o herida (7.9\%), inyectaba tratamiento (6.3\%) o estaba canalizando una arteria o vena (3\%). En la mayoría de las ocasiones el accidente se produjo por una aguja (44.5\%) o una lanceta (22.2\%); la profundidad de la herida fue superficial en el $44.5 \%$ de las personas y sólo en el 12.7\% fue severa (cuadro III).

Una vez ocurrido el accidente, del 100\% de la población de estudio, sólo el 19.1\% del personal se presento al CCIN para su seguimiento a los tres meses, 7.9\% lo hizo a los 6 meses, y sólo el 3.2\% lo concluyó al año; de éste último, el personal era de enfermería e intendencia (cuadro IV). 


\section{DISCUSIÓN}

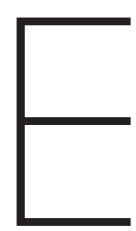

I turno en que se presentan con mayor frecuencia los accidentes por RP fue el matutino, y aunque no se encuentra evidencia en la literatura se puede atribuir a que el número de personal de salud y el número de procedimientos o cuidados es mayor en dicho horario, lo que da la pauta para futuras investigaciones integrando otra variable que tome en cuenta este punto, ya que Neves ${ }^{10}$ ha descrito que la comunicación, la sobrecarga de trabajo, la estructura física, la accesibilidad a los equipamientos de protección, aspectos organizacionales y administrativos pueden considerarse como barreras que interfieren en la seguridad y protección individual.

El personal que tuvo con mayor frecuencia accidentes por RP fue el grupo de enfermería, posiblemente por ser el grupo de trabajo que más horas pasa con los pacientes en los cuales se realizan procedimientos de alto riesgo, estos resultados son semejantes a lo encontrado en algunos estudios, 11,12,13,14 además de que la edad y el tiempo en el servicio son factores que estuvieron ampliamente relacionados con la ocurrencia o no de accidentes, sin embargo, es un dato que no se tuvo en el estudio y esta variable es relevante para normar conductas en la prevención al considerar al personal de nuevo ingreso quien presenta el mayor riesgo.

En este estudio, el servicio donde se presentan con mayor frecuencia los accidentes por RP es en el área no quirúrgica, es decir, en servicios de hospitalización y cuidados críticos, datos que difieren

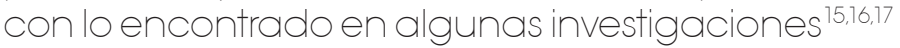
donde es el servicio de quirófano; esta diferencia se puede atribuir al tipo de pacientes que se reciben en un estatus de urgencia, las características clínicas de los pacientes que requieren de una atención inmediata en un menor tiempo posible, el mayor número de punciones y el posible exceso de confianza del personal de salud durante la realización de sus procedimientos, lo que probablemente incremente el riesgo de una punción.

De acuerdo a la evidencia, 12,15 se identificó que la mayoría de los accidentes con punzocortantes que ocurren en el personal de salud está caracterizado por presentarse con mayor frecuencia durante la realización de procedimientos invasivos (como la instalación del acceso vascular, la administración de medicamentos, suturar, realizar una flebotomía) que en aquellos no invasivos, tal y cómo se encontró en nuestro estudio. Por lo que se hace necesario que se normen e implementen dispositivos de seguridad con la finalidad de reducir las lesiones con punzocortantes.

El instrumento con el cual se producen los accidentes, en la mayoría de los casos, está relacionado con los procedimientos invasivos y el área o servicio donde se otorgan la atención (hospitalización, urgencias o quirófano), y entre los más comunes se encuentran las agujas de sutura, las hojas de bisturí y las jeringas según Moreno, ${ }^{16}$ Jagger ${ }^{17}$. Watt ${ }^{18}$ y Gutiérrez ${ }^{19}$. Estos datos concuerdan con nuestros resultados, además de que la mayoría fueron lesiones superficiales, mientas que Tipple ${ }^{12}$ refirió heridas graves con dispositivos que se habían instalado previamente y estaban en contacto con sangre contaminada. Ante esta problemática, se requiere de dispositivos de seguridad para agujas y bisturís, doble enguantado, el uso de agujas de sutura romas y el uso de complementos de sutura, estrategias que han mostrado ser eficaces en la reducción de lesiones por punzocortantes.,20,21,22

Varios autores $2,74,76,23$ han identificado que las medidas adoptadas por el personal de salud después de un accidente o a la exposición con fluidos

50 IMN /W4w Vol.74. No. 7 enero-abril 2075 
corporales se limita a la asistencia local con agua y jabón (53,4\%), no realizan una evaluación médica, hay quien induce el sangrado de la lesión, la mayoría no comunica el accidente laboral al área correspondiente y menos de la mitad no lleva el seguimiento serológico de sí mismo y del paciente, esta evidencia es semejante a lo encontrado en el INClCh, excepto que el personal de salud no refiere si induce o no el sangrado de la lesión, por lo tanto, el personal sabe cómo iniciar el protocolo de vigilancia epidemiológica y reportan el caso al CCIN, pero sólo el 2\% concluye el seguimiento al año.

\section{CONCLUSIONES}

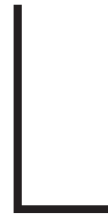

os registros de accidentes laborales son más frecuentes en el personal de enfermería, al ser el profesional que realiza el mayor número de actividades invasivas directamente con el paciente. Los RP causantes de accidentes en su mayoría son agujas, lo que conlleva a reforzar las medidas preventivas encaminadas a eliminar este tipo de lesiones a través de la retroalimentación continua al trabajador de la salud en cuanto a las medidas de bioseguridad, ya que la percepción de la importancia de barreras puede actuar como obstáculo para la adopción de medidas y comportamiento recomendado en procedimientos de riesgo. Con este estudio se observó que el instrumento con el que se realiza la entrevista al personal afectado por punciones carece de ítems para el análisis de factores de riesgo que puede tener relación con las causas de los accidentes y tomar acciones encaminadas a la prevención.

Se desconocen las causas directas que el personal tiene para no reportar el accidente y dar el seguimiento con serología ya que no se da seguimiento al proceso y, por lo tanto, no se ha documentado si existe seroconversión, por lo que se realizan estrategias que recuerde al trabajador su seguimiento hasta cumplir un año del accidente siendo este un tema crucial en la seguridad del personal de salud y pacientes dejando una puerta abierta a continuar con la búsqueda de respuesta a estas interrogantes a través de investigación cuantitativa y cualitativa tomando como referencia los datos arrojados en este estudio.

Cuadro I. Clasificación de factores de riesgo para VIH.

\begin{abstract}
Alto riesgo
Agujas contaminadas con sangre proveniente de pacientes con SIDA en fase terminal que haya producido punción profunda en la piel.
\end{abstract}

Mediano riesgo

Con agujas de sutura, $u$ otras que produzcan lesiones no profundas.
Bajo riesgo

Al contacto con piel y mucosas integras.

Fuente: Tomado de Malagón LG, Álvarez MC. Infecciones Hospitalarias. 3rd ed. México: Editorial Medica Panamerinaca; 2010 
Cuadro II. Clasificación de factores de riesgo hepatitis B, hepatitis C y VIH.

\begin{tabular}{|c|c|c|c|c|c|}
\hline Infección & $\begin{array}{l}\text { Modos de } \\
\text { transmisión }\end{array}$ & $\begin{array}{l}\text { Riesgo de } \\
\text { transmisión a } \\
\text { un huésped } \\
\text { susceptible. } \\
\text { Personal al } \\
\text { paciente }\end{array}$ & $\begin{array}{l}\text { Riesgo de } \\
\text { transmisión a } \\
\text { un huésped } \\
\text { susceptible. } \\
\text { Paciente al } \\
\text { personal }\end{array}$ & $\begin{array}{l}\text { Prevención } \\
\text { primaria } \\
\text { (antes de la } \\
\text { exposición) }\end{array}$ & $\begin{array}{l}\text { Prevención } \\
\text { primaria } \\
\text { (después de la } \\
\text { exposición) }\end{array}$ \\
\hline Hepatitis B & $\begin{array}{l}\text { Vía percutánea, } \\
\text { contacto de sangre } \\
\text { con mucosa y piel } \\
\text { no intacta, semen, } \\
\text { secreciones vaginales } \\
\text { y otros fluidos } \\
\text { corporales }\end{array}$ & Bajo & $\begin{array}{l}\text { Moderado (riesgo } \\
\text { de punción es de } \\
6 \% \text { a } 35 \% \text { ) }\end{array}$ & $\begin{array}{l}\text { Vacuna contra } \\
\text { hepatitis B. Manejo } \\
\text { seguro de punzo- } \\
\text { cortantes, uso de } \\
\text { barreras. Lavado } \\
\text { de manos. }\end{array}$ & $\begin{array}{l}\text { Usar inmunoglobulina } \\
\text { específica contra } \\
\text { hepatitis B e } \\
\text { inmunización en } \\
\text { exposiciones } \\
\text { significativas en } \\
\text { personal susceptible }\end{array}$ \\
\hline Hepatitis C & Similar a hepatitis B & Raro & $\begin{array}{l}\text { Bajo (riesgo de } \\
\text { punción entre 1\% } \\
\text { y } 7 \% \text { ) }\end{array}$ & $\begin{array}{l}\text { Manejo seguro de } \\
\text { punzocortantes, } \\
\text { uso de barreras. } \\
\text { Lavado de manos. }\end{array}$ & $\begin{array}{l}\text { No hay medidas } \\
\text { con suficiente } \\
\text { evidencia. Segui- } \\
\text { miento cercano } \\
\text { con pruebas } \\
\text { serológicas y } \\
\text { enzimas hepáticas. }\end{array}$ \\
\hline VIH & $\begin{array}{l}\text { Principalmente por } \\
\text { contacto percutáneo } \\
\text { con sangre, contacto } \\
\text { de sangre con } \\
\text { mucosas o piel no } \\
\text { intacta. El semen, } \\
\text { secreciones vaginales } \\
\text { u otros fluidos } \\
\text { tienen menor } \\
\text { capacidad para } \\
\text { transmitirlo. }\end{array}$ & Muy raro & $\begin{array}{l}\text { Raro (riesgo de una } \\
\text { punción } 0.3 \% \text { ) }\end{array}$ & $\begin{array}{l}\text { Manejo seguro de } \\
\text { punzocortantes, } \\
\text { uso de barreras. } \\
\text { Lavado de manos. }\end{array}$ & $\begin{array}{l}\text { Seguir guías con } \\
\text { uso de medicamen- } \\
\text { tos antirretrovirales } \\
\text { de acuerdo con el } \\
\text { grado de } \\
\text { exposición }\end{array}$ \\
\hline
\end{tabular}


Cuadro III. Características generales del personal de salud y de los accidentes laborales por residuos punzocortantes.

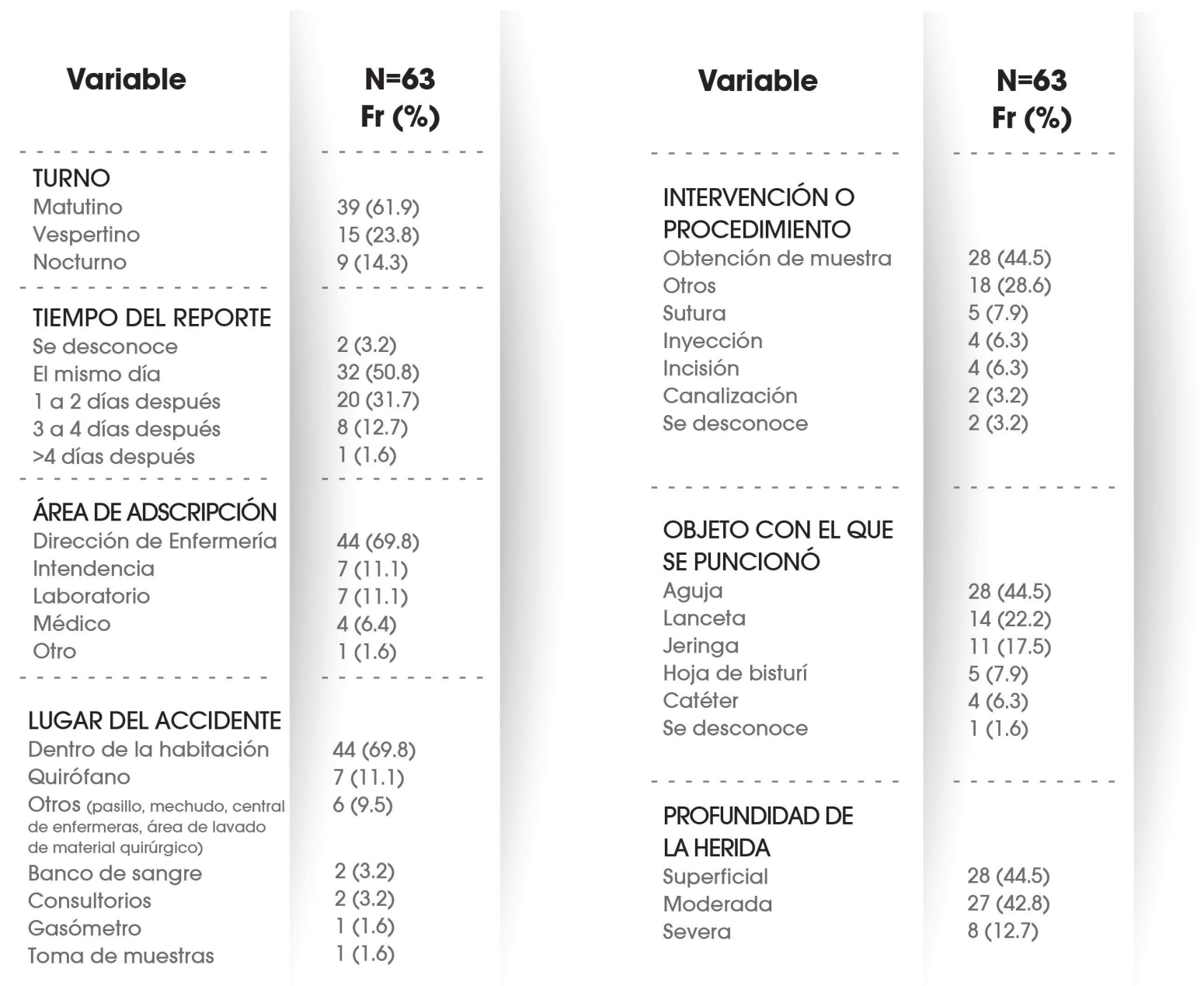

Cuadro IV. Seguimiento del personal de salud que sufrió un accidente laboral por residuos punzocortantes de acuerdo a la ocupación.

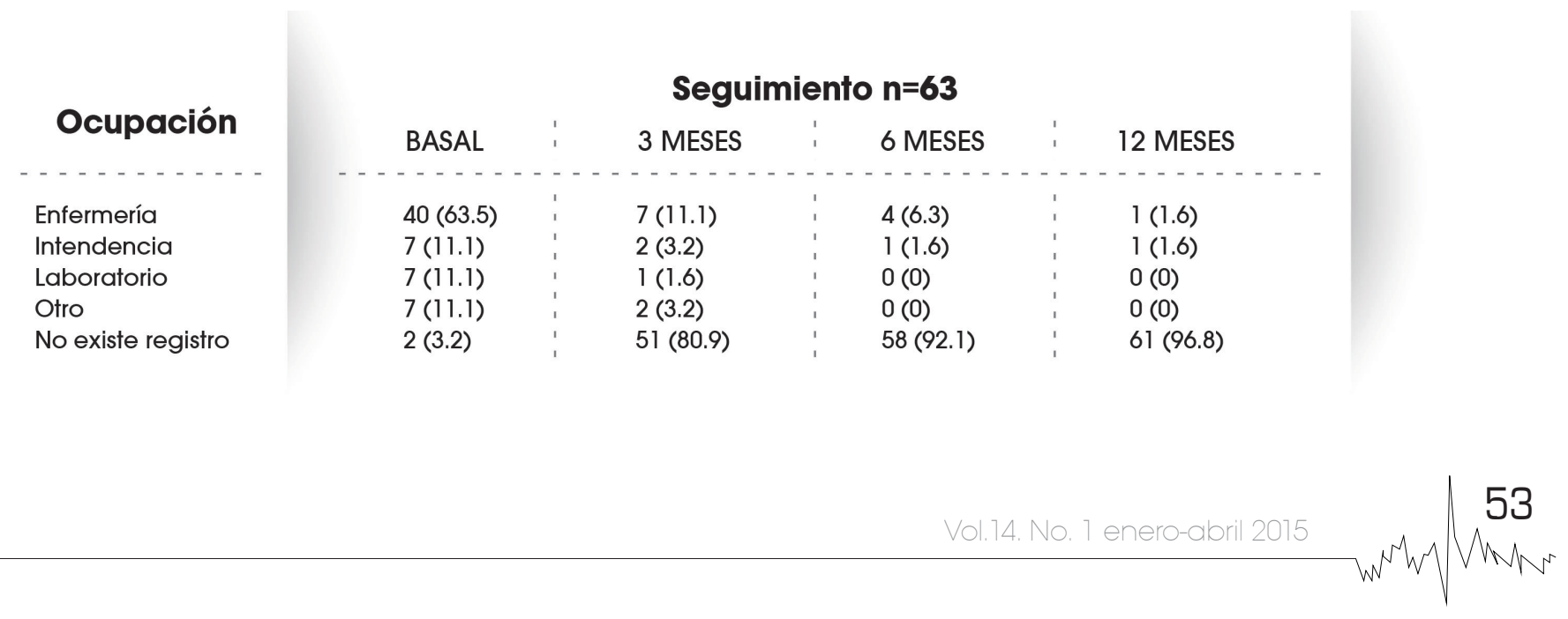




\section{REFERENCIAS BIBLIOGRÁFICAS}

1. Malagón LG, Álvarez MC. Infecciones Hospitalarias. 3rd ed. México: Editorial Medica Panamerinaca; 2010.

2. Luiselli FC, Enriquez RE. NOM-087-ECOL-SSA 1-2002, Protección Ambiental-Salud Ambiental-Residuos peligrosos biológico-infecciosos-Clasifícación y especifícaciones de manejo.Disponible en: http://www. salud.gob.mx/unidades/cdi/nom/087ecolssa.html

3. Guauche GH, Menéndez MN, Piñeira CSA, Morales PC, Feesneda SG, Gutiérrez GF. Riesgo ocupacional por exposición a objetos punzocortantes en trabajadores de la salud. Medicrit. 2006; 3(2): 56-60.

4. Ghany MG, Strader DB, Thomas DL, Seeff LB. Diagnosis, management, and treatment of hepatitis C. Hepatology. .(serial on the internet). 2009 Apr; \{cited 02 January 2014\} 49(4): 1335-74. Available from: http://www.ncbi. nlm.nih.gov/pubmed/19330875

5. Brooks G, Butel J, Morse S. Microbiología médica. 18a ed. México: Manual Moderno; 2010.

6. Ponce de León RS, Soto HJ. Infecciones intrahospitalarias. México: Interamericana; 1996.

7. Manual Administrativo del Comité de Control de Infecciones Nosocomiales del Instituto Nacional de Cardialgia キlgnacio Chávez . 2010.
8. Cámara de Diputados del H. Congreso de la Unión. Ley General de Salud. (Internet). México: Diario OÏcial de la Federación: 2012. (consultado 15 enero 2013). Disponible en: http://www.salud.gob.mx/unidades/ coli/nom/compi/rlgsmis.html

9. Complete transcript 01 the Nuremberg Medical Trial: United States v. Karl Brandt et al. En: Colomer MF, Pastor GLM. ÕVigencia del Código de Nüremberg después de cincuenta años? Cuad Bioética (Internet). 1999 ene-mar;IX(37): 103-1 12. Disponible en: http:// aebioetica.org/revistas/1999/1/37/07-2-bioetica-37.pdf

10. Neves HCC, Souza ACS, Medeiros M, Munari DB, Ribeiro LCM, Tipple AFV. La seguridad de los trabajadores de enfermería y los factores determinantes para adhesión a los equipamientos de protección individual. Rev. Latino-Am. Enfermagem. (Internet) mar.-abr. 2011 (Citado 8 ene 2014); 19(2):(aprox. 09 pantallas). Disponible en: www.eerp.usp.br/rlae

11. Núñez ZL, Ramírez DR. Características epidemiológicas de los accidentes laborales punzocortantes y de exposición mucocutánea en el personal asistencial de Enfermería del Hospital Alberto Sabogal Sologuren Es Salud. Rev. Cient. Enffi Recién. 2002. 37-42.

12. Tipple AFV, Silva EAC, Teles SJ, Mendonça KM, Souza ACS, Melo DS. Acidente com material biológico no atendimento pré-hospitalar móvel: realidade para 
trabalhadores da saúde e não saúde. Rev Bras Enferm, Brasilia 2013 mai-jun; 66(3): 378-84.

13. Morales-Aguirre JJ. Frecuencia y mecanismos de exposición accidental a productos biológicos potencialmente infecciosos en personal de salud. Bol Med Hosp Infant Mex. jul-agos 2006; 63(4): 247-54.

14. Vieira M, Padilha MI, Pinheiro RDC. Análisis de los accidentes con material biológico en trabajadores de la salud. Rev. Latino-Am. Enfermagem. (Internet) mar.-abr. 2011 (Citado 8 ene 2014); 19(2):(aprox. 09 pantallas). Disponible en: www. eerp.usp.br/rlae

15. Barroso-Aguirre J, Pimentel-Nieto D, Morales-Carmona F, Cosme-Pérez J, Santillán-Palomo V, Rivas-Torres $M P$, et al. Heridas con material punzocortante en un Instituto Nacional de Salud de México. Perinatol Reprod Hum. 2009; 23(3): 141-9.

16. Moreno RB, Barreto AR, Mora MA, Morales ZM, Rivas PF. Accidentes biológicos por exposición percutánea y contacto cutáneo-mucoso en el personal de enfermería del Instituto Autónomo Hospital Universitario de Los Andes, Mérida, Venezuela, 2003. Revista Facultad Nacional de Salud Pública. Ene-jun 2004; 22 (1): 73-86.

17. Jagger J, Berguer R, Phillips EK, Pärker G, Gomaa $A E$. Increase in sharps injuries in surgical settings versus nonsurgical settings after passage of national needlestick legislation. J Am Coll Surg 2010; 210: 496-502.
18. Watt AM, Patkin M, Sinnott MJ, Black RJ, Maddern GJ. Scalpel safety in the operative setting: a systematic review. Surgery 2010; 147: 98-106.

19. Gutiérrez C, Alarcón J, Sánchez S, Carrión M. Prevalencia y factores asociados a heridas punzo-cortantes en trabajadores de salud del primer nivel de atención. Dirección de Salud V Lima Ciudad, 2005. Rev. Peru. Epidemiol. (Internet) Agos 2008 (citado 8 de enero de 2014); 12(2): (aprox. 9 plantillas). Disponible en: $h$ ttp:// rpe.epiredperu.net/v12_n02_2008.html

20. DeGirolamo KM, Courtemanche DJ, Hill WD, Kennedy A, Skarsgard EDUse of safety scalpels and other safety practices to reduce sharps injury in the operating room: What is the evidence? Can J Surg, Aug 2013; 56(4): 263-9.

21. Bell PR, McNicholl BP. The needle catcher fl a safer way to suture. Surgeon 2009; 7: 251-2.

22. Stringer B, Haines T, Goldsmith $\mathrm{CH}$, Blythe J, Berguer $\mathrm{R}$, Andersen J, et al. Hands-free technique in the operating room: reduction in body luid exposure and the value of a training video. Public Health Rep. 2009; 124 (Suppl 1): 169-79.

23. Paiva MHRS, Oliveira AC. Fatores determinantes $e$ condutas pós-acidente com material biológico entre prö̈sisonais do atentimento pré hospitalar. Rev Bras Enferm, Brasília 2011 mar-abr; 64(2): 268-73. 